\title{
3 Research Square

\section{Characterization of interactions between the soybean plasma membrane- intrinsic proteins GmPIP1s and GmPIP2s responding to salt stress}

Weicong Qi ( $\nabla$ weicong_qi@163.com )

Jiangsu Province Academy of Traditional Chinese Medicine https://orcid.org/0000-0001-5459-2767

Jia Liu

Jiangsu Academy of Agricultural Sciences

Dayong Zhang

Nanjing Agricultural University

Haiying Lu

Jiangsu Academy of Agricultural Sciences

Hongbo Shao

Jiangsu Academy of Agricultural Sciences

Qun Wan

Jiangsu Academy of Agricultural Sciences

Yihong Huang

Jiangsu Academy of Agricultural Sciences

Research article

Keywords: Gene expression pattern, GmPIPs, Protein interactions, Salt stress, Soybean

Posted Date: November 2nd, 2020

DOI: https://doi.org/10.21203/rs.3.rs-80161/v1

License: (c) (1) This work is licensed under a Creative Commons Attribution 4.0 International License.

Read Full License 


\section{Abstract}

Background: Salt tolerance is a key trait in soybean breeding and plant responses to salt stress include physiological and biochemical changes that affect the movement of water across the plasma membrane. In this study, we report the interactions of a set of aquaporins, soybean (Glycine max) plasma membraneintrinsic proteins (GmPIPs), in response to salt stress.

Results: GmPIP1;5 and GmPIP1;6 formed hetero-tetramers with GmPIP2;4, GmPIP2;6, GmPIP2;8, GmPIP2;9, GmPIP2;11, and GmPIP2;13. We detected interactions between GmPIP1;6 and GmPIP1;7, but not between GmPIP1;6 and GmPIP1;5. Furthermore, GmPIP2;9 formed homo-tetramers, and this interaction was strengthened under salt and osmotic stress. Expression analysis indicated complex and unique responses to salt stress depending on the duration of the stress. For example, GmPIP2;8, encoding one of the heteromer-forming PIP proteins, was highly up-regulated under early salt stress.

Conclusions: Our study highlights the vital role of hetero- and homo-tetramers, in salt tolerance; and improves understanding of the mechanisms by which soybean aquaporin isoforms respond to abiotic stress.

\section{Background}

Plasma membrane intrinsic proteins (PIPs) are aquaporins that localize to the plasma membrane and regulate the flow of water and solutes through membranes. PIPs cluster into two evolutionary subgroups, PIP1 and PIP2. The copy numbers of the PIP1 and PIP2 isoforms vary among species: there are five PIP1s and four PIP2s in Arabidopsis thaliana[1], eight PIP1s and fourteen PIP2s in soybean (Glycine max, Zhang et al. 2013)[2] and Brassica rapa [3], and four PIP1s and five PIP2s in chickpea (Cicer arietinum L.) [4].

Plant PIPs contain two conserved domains: transmembrane domains (TMDs) and NPA motifs (asparagine-proline-alanine, NPA). Moreover, plant PIPs vary in the lengths of their $\mathrm{N}$ - and $\mathrm{C}$-terminal ends (Yaneff et al. 2015)[5]. Previous biochemical and crystallography-based 3D structural analyses reported that PIPs assemble as hetero- or homo-tetramers (Murata et al. 2000; Gonen et al. 2004; Beesesims et al. 2011)[6-8]. Moreover, assays in Xenopus oocytes suggested that PIP1 and PIP2 coexpression could help PIP1 reach the plasma membrane (PM) and increase the permeability coefficient $\left(P_{f}\right)$ in comparison with expressing PIP2 alone (Fetter et al. 2004; Bienert et al. 2012). These results imply that PIP1 and PIP2 regulate several physiological processes jointly and may form functional units that aid solute transport (Yaneff et al. 2015)[5].

PIP1 and PIP2 form a complex that regulates water transport [9-11]. Previously, Harvengt et al. [12] isolated two isoforms of aquaporin from the protein-storage vacuoles of Lens culinaris Med. seeds. Using chemical cross-linking, they found that both isoforms belonged to the same oligomer in the membrane. Co-expression of maize (Zea mays) ZmPIP1;2 with ZmPIP2;1, ZmPIP2;4, or ZmPIP2;5 led to an increase in $P_{f}[13]$. Moreover, the interaction between PIP proteins enhanced the delivery of PIP2 from the Golgi to 
the plasma membrane [14]. Additionally, in the extremophile Thellungiella halophila, the interaction between PIP1 and PIP2 triggered multiple physiological responses when the plant was exposed to salt stress [15]. When co-expressed in Xenopus oocytes, Beta vulgaris BVPIP1;1 and BVPIP2;2 enhanced PM water permeability [16], but $B V P I P 2 ; 1$ did not bind $B V P I P 1 ; 1$ or transport it to the oocyte PM[17]. Thus, the phenomenon of PIP interaction is specific, and not conserved in all isoforms.

Protein-protein and protein-macromolecule interactions play crucial roles in maintaining various physiological activities and molecular functions in plants. Salt stress is an important abiotic stressor of soybean that disrupts many physiological processes and reduces potential yield. We hypothesized that physical interactions between PIPs are a key component of the soybean response to salt stress. To detect these interactions, we carried out yeast two-hybrid assays. Our results show hetero- and homotetramerization of PIPs, and changes in PIP gene expression, which might affect water channel activity and plant salt tolerance.

\section{Results}

\subsection{Sequence analysis of soybean GmPIPs}

Soybean GmPIPs contain six TMDs and two conserved NPA motifs. Even though the transmembrane regions of both types of PIPs share high similarity, we found several differences in the amino acid sequences of TMD2 and TMD4 (Supplementary Fig. 1). The structural differences between PIP1 and PIP2 are limited to their $\mathrm{N}$ and $\mathrm{C}$ terminal ends (Yaneff et al., 2015) [5]. In soybean, the N-terminal ends of GmPIP1s are longer than those of GmPIP2s by about 15 amino acids. However, the C-terminal ends of GmPIP1s are shorter than those of GmPIP2s by about 8 amino acids (Supplementary Fig. 1).

Phylogenetic analysis indicated that the GmPIP1s and the GmPIP2s formed two separate clusters (Supplementary Fig. 2). The clusters remained even after the addition of PIPs from other species, such as rice (OsPIPs) and Arabidopsis (AtPIPs), to the phylogenetic analysis (Supplementary Fig. 3).

We downloaded and analyzed whole genome duplication data for soybean from PGDD (http://chibba.agtec.uga.edu/duplication/). We found variation in segmental-duplication between some of the isoforms (Reference to Table or Figure): among GmPIP1;1, GmPIP1;2, GmPIP1;7 and GmPIP1;8; GmPIP1;3, GmPIP1;4, GmPIP1;5 and GmPIP1;6; GmPIP2;1 and GmPIP2;2; GmPIP2;3, GmPIP2;4, GmPIP2;5, GmPIP2;6 and GmPIP2;8; GmPIP2;7, GmPIP2;4, GmPIP2;6, GmPIP2;8, GmPIP2;13, GmPIP2;14, and $G m P I P 1 ; 8, G m P I P 2 ; 13, G m P I P 2 ; 9, G m P I P 2 ; 10, G m P I P 2 ; 11$ respectively, however, tandem-duplication variation happened only in GmPIP2;10 and GmPIP2;11.

\subsection{Hetero- and homo-tetramerization in GmPIP1s and GmPIP2s}

Based on our phylogenetic analysis, we selected some (Supplementary Fig. 3) of the GmPIPs for yeast two-hybrid assays. A total of 255 PIP1-PIP1, PIP1-PIP2, and PIP2-PIP2 combinations were tested. We found that GmPIP1;5 and GmPIP1;6 could each interact with GmPIP2;2, GmPIP2;4, GmPIP2;6, GmPIP2;8, GmPIP2;9, GmPIP2;11, and GmPIP2;13 (Fig. 1). Among the PIP1-PIP1 pairs tested, we found an 
interaction only between GmPIP1;6 and GmPIP1;7 (Fig. 1). In addition, we observed that salt treatment strengthened most of the interactions, but osmotic stress induced by mannitol treatment did not (Fig. 2). Furthermore, we found that only GmPIP2;9 could form homo-tetramers, and that this interaction was enhanced by salt stress, but weakened by osmotic stress (Fig. 3).

2.3 The transcriptional profiles of GmPIPs under salt stress

Two-week old plants were subjected to salt stress $(200 \mathrm{mM} \mathrm{NaCl})$ for $0 \mathrm{~h}, 2 \mathrm{~h}$, and $12 \mathrm{~h}$, and the transcriptional profile of GMPIP genes was examined. The expression levels of three GMPIP genes (GmPIP1;3, GmPIP1;4, and GmPIP2;8) were significantly elevated in response to salt stress (Fig. 4). Of particular note was GmPIP2;8, whose transcript levels were about 6 times higher after $12 \mathrm{~h}$ of salt stress. Most of the other PIP genes were down-regulated after treatment for $12 \mathrm{~h}$, except for GMPIP2;7 (not shown in the figure). While the expression levels of five GmPIP genes (GMPIP1;1, GmPIP1;7, GmPIP2;3, GmPIP2;5, and GmPIP2;8) were lowered immediately in response to salt stress, the levels of others (such as GmPIP1;6, GmPIP2;13, and GmPIP2;1) decreased gradually. Furthermore, the transcript levels of GmPIP1;5, GmPIP1;8, and GmPIP2;2 did not change even after $12 \mathrm{~h}$ of salt stress. The transcript levels of GmPIP1;2 and GmPIP2;4 decreased significantly at $2 \mathrm{~h}$ and then recovered at $12 \mathrm{~h}$ to the same level as at 0 h. By contrast, the transcript levels of four GmPIP genes (GMPIP2;1, GmPIP2;9, GmPIP2;10, and GmPIP2;11) increased at $2 \mathrm{~h}$, and then sharply decreased afterwards (Fig. 4). Therefore, the GmPIP genes show different transcript profiles in response to salt stress.

\section{Discussion}

Numerous studies have reported the identification of functional genes that are relevant to plant stress tolerance and that can be used for crop improvement[18-21]. To aid efforts to breed salinity tolerance in soybean, we analyzed the orthologs of plasma membrane intrinsic proteins (PIPs) in soybean and tested their interactions using a yeast two-hybrid system. The interaction between PIP1 and PIP2 proteins functions as the main signal for cell membrane water and salt exchange and PIPs have been considered as functional units that perform their physiological roles under different environmental stresses, such as salt and drought stress. Aquaporins play an important role in growth regulation of plants by influencing root water uptake and leaf gas exchange. The soybean PIP1, GmPIP1;6, has previously been well characterized. Its function in growth regulation and salt tolerance was analysed by constitutive overexpression [22]. A separate study highlighted the involvement of several aquaporin homologs in response to a variety of environmental stressors that interrupt plant cell osmotic balance [23].

PIP1 and PIP2 have highly conserved peptide sequences, and the main differences between them are the lengths of their $\mathrm{N}$ and $\mathrm{C}$ terminal ends [5]. In soybean, the $\mathrm{N}$ terminal ends of GmPIP1 are longer than that of GmPIP2 (approximately 15 amino acids), however, the $C$ terminal ends of GmPIP1 type are shorter than that of GmPIP2 type (approximately 8 amino acids). Interestingly, the transmembrane region of both GmPIP1 and GmPIP2 nearly share the same section (Supplementary Figure 1). 
Ispolatov et al. [24] proposed that duplicated proteins were more likely to interact among themselves than with other proteins, and that paralogous interactions were inherited from ancient homo-dimeric proteins, rather than established de novo after gene duplication. In evolution progress, gene duplication events increase gene number by tandem- and segmental-duplication [25]. Soybean (Glycine max (L.) Merr.) is an important crop and well-studied. Previous research reported that soybean is a paleopolyploid, and at least two rounds of large-scale duplication occurred in its ancestral genome at approximately 14- to 42- million ago [26]. In this study, we searched the database PGDD (http://chibba.agtec.uga.edu/duplication/), and found many duplication events in the GmPIP family. Expansion of aquaporin gene families via genome duplication events have been reported in other plants [27].

The transcriptional profiles of PIPs may provide evidence for PIP1-PIP2 interactions. For example, a joint increase (or decrease) in the expression of specific PIP1-PIP2 pairs in plants under stress may indicate shared functionality. Thus, the formation of hetero-tetramers composed of specific PIP1s and PIP2s could be affected by their mRNA abundance [5]. Transcriptional profiles in rice, maize, and Arabidopsis indicate interactions between PIP1-PIP2 pairs in these species [5]. Additionally, Zargar et al. [28] developed a gene co-expression network of rice aquaporin genes (OsPIPs) and tonoplast intrinsic proteins (OSTIPS) using Rice Friend server (http://ricefrend.dna.affrc.go.jp). They found co-expression of PIP1-PIP2 pairs, indicating likely physical interaction between these proteins. In this study, we found physical interactions among GmPIPs using yeast two-hybrid assays. We detected both homotetramers and heterotetramers among these proteins. Salt, but not mannitol, enhanced these interactions. Only GmPIP2;9 could form homotetramers, and this interaction was enhanced by salt stress, but weakened by osmotic stress. Our results corroborate those of Bienert et al. [29], who reported PIP heterotetramerization under salt stress in Selaginella moellendorffii.

The TMDs of GmPIPs were predicted by SMART software. All GmPIPs contained six TMDs. GmPIP1;5 and GmPIP1;6 differed by two amino acids in TMD2 (D/Y) and TMD6 (H/Q). However, there were many differences in TMD2, TMD4, and TMD6 among GmPIP2;4, GmPIP2;6, GmPIP2;8, GmPIP2;9, GmPIP2;10, and GmPIP2;11. This indicates that the highly conserved sequences in TMD1, TMD3, and TMD5 may play a crucial role in the formation of PIP1-PIP2 pairs between GmPIP1;5 and GmPIP1;6, and among GmPIP2;4-GmPIP2;11. Using extensive amino acid substitution mutagenesis, Yoo et al. (2016) studied tetramer formation in ArabidopsisAtPIP2;1. They demonstrated that TMD1, TMD2, and TMD5 contained essential amino acid residues key to tetramer formation.

In addition, the expression profiles of GMPIPs under salt stress also showed similar expression patterns in GmPIP1;5 and GmPIP1;6, and in GmPIP2;3, GmPIP2;4, GmPIP2;5, GmPIP2;6; GmPIP2;9, GmPIP2;10, GmPIP2;17; GmPIP2;13 and GmPIP2;14 respectively, which is partly consistent with the sequence similarities and interaction patterns among GmPIPs. The expression of GmPIP1;3, GmPIP1;4, GmPIP2;1, GmPIP2;8, GmPIP2;9, GmPIP2;10, GmPIP2;11 was significantly up regulated by salt stress. However, the other GmPIPs were significantly downregulated by salt stress, except GmPIP1;9, 1;10, 2;7 and 2;12. 
Aquaporins are implicated in a variety of stress responses that disturb plant cell osmotic balance and nutrient homeostasis (Yaneff et al. 2015)[5]. They are involved in the Arabidopsis response to drought stress (Afzal et al., 2016)[23], in leaves and roots of sugar beet under salt stress (Lv et al., 2018)[30], and in rice tolerance to salt stress and cold stress (Qiang et al. 2015)[15]. We have discovered that some GmPIPs are significantly up- or down-regulated in response to salt stress. GmPIP1;6 is the closest ortholog to ArabidopsisAtPIP1;2 (with an amino acid sequence identity of $84.8 \%$ ), which localizes to the Golgi apparatus and the membrane system. AtPIP1;2 was considered as a functional water channel when it was expressed alone in Xenopus oocytes [31]. A previous study also suggested that many GmPIPs have high sequence similarity but diverse functions (Zhang et al. 2013)[2]. GmPIP1;6 could interact with other GmPIP2 type aquaporins, which implied they may play crucial roles in aquaporin trafficking from the Golgi apparatus to the membrane system in plants. The functions and molecular mechanisms of the diverse families of plant PIPs still need further study.

\section{Conclusions}

In this study we identified the PIP gene families in the soybean genome (GMPIP) and analyzed the expression patterns of GmPIPs under salt stress and interactions among the encoded proteins. We found that $G m P I P 1 ; 5$ and GmPIP1;6 each formed hetero-tetramers with six GmPIP2-type aquaporins. GmPIP1;6 interacted with GmPIP1;7. Furthermore, GmPIP2;9 formed homo-tetramers. These interactions were strengthened by salt stress, but not by osmotic stress. Most of the genes encoding interacting GmPIPs exhibited a similar expression pattern under salt stress. Our results improve our understanding of the interactions among soybean PIP isoforms and the role of homo- and hetero-tetramers in the response to salt stress.

\section{Abbreviations}

Glycine max - Gm; plasma membrane-intrinsic protein - PIP, transmembrane domains -TMD; plasma membrane - PM; permeability coefficient - $\mathrm{P}_{f}$; Zea mays $-Z m$; stand derivation - SD.

\section{Methods}

\subsection{Soybean plant materials and treatments}

The seeds of soybean variety Willimas82 (Glycine max) were from the seed stock of our lab, and they were grown in pots for the salt treatment. Two-week-old seedlings were placed into $1 / 2$ Hoagland solution for two days as an adjustment process, then subjected to $200 \mathrm{mM} \mathrm{NaCl}$ solution made with $1 / 2$ Hoagland solution for 12 hours and the treatment with $1 / 2$ Hoagland solution as a control respectively, all the treated seedlings were kept in a growth chamber with a temperature of $24^{\circ} \mathrm{C}$.

\subsection{Bioinformatics analysis of soybean PIPs}


To analyze the duplication events among soybean GmPIPs, the data were downloaded from http://chibba.agtec.uga.edu/duplication/ and the tandem and segmental duplication of GmPIPs were found out respectively. The transmembrane region for GmPIPs was predicted using http://smart.emblheidelberg.de/ online software. The evolutional trees for GmPIPs and other plant species PIPs proteins, downloaded from http://ricefrend.dna.affrc.go.jp, were constructed using MEGA5.0 software [32]. The multiple alignment was performed using Cluxtal $\mathrm{X}$ and GeneDoc.

\subsection{RNA extraction and cDNA synthesis}

Total RNA was extracted from the root tissue as well as other samples of the seedlings subjected to 200 $\mathrm{mM} \mathrm{NaCl}$ for 0 (CK), 2 and $12 \mathrm{~h}$, respectively, with Promega RNA extraction kit. After the DNA removal through DNase I digestion, RNA quality and integrity was detected using $1.2 \%$ agarose gel electrophoresis. The cDNA synthesis was performed according to the procedure of the reverse transcription kit (HaoJia Technology Development Co., Ltd. Shanghai, China).

\subsection{GmPIPs gene cloning and constructs preparation}

Full-length ORF of GmPIPs was amplified from the root or leaf tissues of soybean (variety Willimas 82) seedlings using primers containing enzyme digestion sites (Supplementary Table 1). PCR products were transferred to the pGEM-T Easy Vector and the resulting vector pGEM-T-GmPIPs were sequenced by Nanjing TSINGKE Biological Technology Co., Ltd to validate the sequence, every gene repeated three times. The pGADT7, pGBKT7 vectors and pGEM-T-GmPIPs were digested using Ncol and EcoRI, then the digested vector and gene fragments were ligased using T4 DNA ligase enzyme. After transformation, positive clones detection and sequencing validation for pGADT7-GmPIPs and pGBKT7-GmPIPs, and then they were prepared for the following yeast two hybrid assay.

\subsection{Yeast two hybrid assay}

The yeast two-hybridization assay was performed according to the protocol of The Matchmaker ${ }^{\mathrm{TM}}$ Gold Yeast Two-Hybrid System (Clontech).

After the co-transformation of pGADT7-GmPIPs and pGBKT7-GmPIPs, the equal amounts of yeast clones were transferred and plated on selective mediums of SD-Leu-Trp and SD-Leu-Trp-His-Ade+Aba+Xa-gal respectively. They were incubated at $30^{\circ} \mathrm{C}$ until the appearance of distinguishable colonies. Yeast cells having pGBKT7-53 and PGADT7-SV40 plasmids were as positive controls, and those having pGBKT7-Lam and pGADT7-SV40 were the negative. Meanwhile, the yeast clones were also plated on SDLeu-Trp-His-Ade+Aba+X-a-gal with $100 \mathrm{mM} \mathrm{NaCl}$ or $200 \mathrm{mM}$ Mannitol respectively. The $\mathrm{ddd}_{2} \mathrm{O}$ was used for the yeast dilution and then $1 \mathrm{ml}$ of $O D$ and $1 \mathrm{ml}$ of the solution was poured in a cuvette to confirm the OD.

6.6 Gene expression analysis 
Quantitative real-time PCR was performed to assay the expression level of GmPIP genes in response to salt stress. Primers for each gene were listed in Supplementary Table 2. The soybean GmTUBB3 (NM_001252709.2) and GmActin (NM_001289231.1) were used as internal references. All of reactions were carried out with the SYBR® Premix Ex Taq ${ }^{\text {TM }}$ (Takara, China) reaction mixture in a Bio-Rad CFX connect Real-Time system (Bio-Rad Laboratories, California, USA). The experimental conditions were set as: $10 \mathrm{~s}$ at $95^{\circ} \mathrm{C}$, followed by 40 cycles of $5 \mathrm{~s}$ at $95^{\circ} \mathrm{C}$ for denaturing and $30 \mathrm{~s}$ at $60^{\circ} \mathrm{C}$ for annealing and extension. The results were obtained from the relative gene expression levels calculated by the $2^{-} \Delta \Delta \mathrm{Ct}$ method and the plants of $0 \mathrm{~h}$ treatment were as control.

\subsection{Statistical analysis}

The data are the average of two replicates \pm stand derivation (SD) for each treatment. The data was analyzed by ANOVA using SPSS 20.0. Values of significantly difference $(P<0.05)$ are marked by lowercase letters $(a-d)$ in the figures.

\section{Declarations}

\subsection{Ethics approval and consent to participate}

There is no experimental material of animal and human used in present research. Here we declare the experiments and researches have no conflict against ethics at all.

\subsection{Consent to publish}

All the participators involved in present research have thoroughly read the manuscript and approved the submission.

\subsection{Availability of data and materials}

The data and results present in the manuscript are all available and free to be accessed by the readers.

\subsection{Competing interests}

The authors declare that they have no conflict of interest.

\subsection{Funding}

This study was sponsored by Jiangsu Planned Projects for Postdoctoral Research Funds (1601032A), National Natural Science Foundation of China (31600211), Natural Science Foundation of Jiangsu Province (BK20181244); Jiangshu Shuangchuang Talent Plan, the Excellent Scientist Plan of JAAS and Jiangsu Agriculture Science and Technology Innovation Fund [CX(15)1005]. The funding bodies just provided the financial support to present study, while they did not play any role in experiment design, implementation, data analysis, or manuscript writing. 
WQ, JL and DZ conceived, designed, and conducted the experiments. $\mathrm{YH}, \mathrm{QW}$ and $\mathrm{W} \mathrm{Q}$ analysed the data and results. DZ wrote the manuscript. HS and HL monitored the experiments and critically. All authors have read and approved the manuscript.

\section{References}

1. Quigley F, Rosenberg JM, Shachar-Hill Y, Bohnert HJ: From genome to function: the Arabidopsis aquaporins. Genome biology 2001, 3(1):research0001. 0001.

2. Zhang DY, Ali Z, Wang CB, Xu L, Yi JX, Xu ZL, Liu XQ, He XL, Huang YH, Khan IA: Genome-wide sequence characterization and expression analysis of major intrinsic proteins in soybean (Glycine max L.). PLoS one 2013, 8(2):e56312.

3. Kayum MA, Park J-I, Nath UK, Biswas MK, Kim H-T, Nou I-S: Genome-wide expression profiling of aquaporin genes confer responses to abiotic and biotic stresses in Brassica rapa. BMC plant biology 2017, 17(1):23.

4. Deokar AA, Tar'an B: Genome-wide analysis of the aquaporin gene family in chickpea (Cicer arietinum L.). Frontiers in plant science 2016, 7:1802.

5. Yaneff A, Vitali V, Amodeo G: PIP1 aquaporins: intrinsic water channels or PIP2 aquaporin modulators? FEBS letters 2015, 589(23):3508-3515.

6. Murata K, Mitsuoka K, Hirai T, Walz T, Agre P, Heymann JB, Engel A, Fujiyoshi Y: Structural determinants of water permeation through aquaporin-1. Nature 2000, 407(6804):599-605.

7. Gonen T, Sliz P, Kistler J, Cheng Y, Walz T: Aquaporin-0 membrane junctions reveal the structure of a closed water pore. Nature 2004, 429(6988):193-197.

8. Beese-Sims SE, Lee J, Levin DE: Yeast Fps1 glycerol facilitator functions as a homotetramer. Yeast 2011, 28(12):815-819.

9. Liu C, Fukumoto T, Matsumoto T, Gena P, Frascaria D, Kaneko T, Katsuhara M, Zhong S, Sun X, Zhu Y: Aquaporin OsPIP1; 1 promotes rice salt resistance and seed germination. Plant Physiology and Biochemistry 2013, 63:151-158.

10. Mahdieh M, Mostajeran A, Horie T, Katsuhara M: Drought stress alters water relations and expression of PIP-type aquaporin genes in Nicotiana tabacum plants. Plant and Cell Physiology 2008, 49(5):801-813.

11. Vandeleur RK, Mayo G, Shelden MC, Gilliham M, Kaiser BN, Tyerman SD: The role of plasma membrane intrinsic protein aquaporins in water transport through roots: diurnal and drought stress responses reveal different strategies between isohydric and anisohydric cultivars of grapevine. Plant physiology 2009, 149(1):445-460.

12. Harvengt $P$, Vlerick A, Fuks B, Wattiez R, Ruysschaert JM, Homble F: Lentil seed aquaporins form a hetero-oligomer which is phosphorylated by a $\mathrm{Mg}^{2+}$-dependent and $\mathrm{Ca}^{2+}$-regulated kinase. Biochemical Journal2000, 352(1):183-190. 
13. Fetter $\mathrm{K}$, Wilder VV, Menachem $\mathrm{M}$, Chaumont $\mathrm{F}$ : Interactions between plasma membrane aquaporins modulate their water channel activity. Plant Cell 2004, 16(1):215-228.

14. Hachez C, Laloux T, Reinhardt H, Cavez D, Degand H, Grefen C, De Rycke R, Inzé D, Blatt MR, Russinova E: Arabidopsis SNAREs SYP61 and SYP121 coordinate the trafficking of plasma membrane aquaporin PIP2; 7 to modulate the cell membrane water permeability. The Plant Cell 2014, 26(7):3132-3147.

15. QIANG X-j, YU G-h, JIANG L-I, SUN L-I, ZHANG S-h, Wei L, CHENG X-g: Thellungiella halophila ThPIP1 gene enhances the tolerance of the transgenic rice to salt stress. Journal of Integrative Agriculture 2015, 14(10):1911-1922.

16. Bellati J, Alleva K, Soto G, Vitali V, Jozefkowicz C, Amodeo G: Intracellular pH sensing is altered by plasma membrane PIP aquaporin co-expression. Plant molecular biology 2010, 74(1-2):105-118.

17. Jozefkowicz C, Rosi P, Sigaut L, Soto G, Pietrasanta LI, Amodeo G, Alleva K: Loop A is critical for the functional interaction of two Beta vulgaris PIP aquaporins. PLOS One 2013, 8(3):e57993.

18. Wu J, Jiang Y, Liang Y, Chen L, Chen W, Cheng B: Expression of the maize MYB transcription factor ZmMYB3R enhances drought and salt stress tolerance in transgenic plants. Plant physiology and biochemistry 2019, 137:179-188.

19. Pavia I, Roque J, Rocha L, Ferreira H, Castro C, Carvalho A, Silva E, Brito C, Gonçalves A, Lima-Brito J: Zinc priming and foliar application enhances photoprotection mechanisms in drought-stressed wheat plants during anthesis. Plant Physiology and Biochemistry 2019, 140:27-42.

20. Sun $\mathrm{K}$, Wang $\mathrm{H}$, Xia Z: The maize bHLH transcription factor bHLH105 confers manganese tolerance in transgenic tobacco. Plant Science 2019, 280:97-109.

21. Brito C, Dinis L-T, Ferreira H, Rocha L, Pavia I, Moutinho-Pereira J, Correia CM: Kaolin particle film modulates morphological, physiological and biochemical olive tree responses to drought and rewatering. Plant Physiology and Biochemistry 2018, 133:29-39.

22. Zhou L, Wang C, Liu R, Han Q, Vandeleur RK, Du J, Tyerman S, Shou H: Constitutive overexpression of soybean plasma membrane intrinsic protein GmPIP1; 6 confers salt tolerance. BMC Plant Biology 2014, 14(1):181.

23. Afzal Z, Howton T, Sun Y, Mukhtar MS: The roles of aquaporins in plant stress responses. Journal of developmental biology 2016, 4(1):9.

24. Ispolatov I, Yuryev A, Mazo I, Maslov S: Binding properties and evolution of homodimers in proteinprotein interaction networks. Nucleic acids research 2005, 33(11):3629-3635.

25. Bancroft I: Duplicate and diverge: the evolution of plant genome microstructure. TRENDS in GeneticS 2001, 17(2):89-93.

26. Shoemaker RC, Schlueter J, Doyle JJ: Paleopolyploidy and gene duplication in soybean and other legumes. Current opinion in plant biology 2006, 9(2):104-109.

27. Abascal F, Irisarri I, Zardoya R: Diversity and evolution of membrane intrinsic proteins. Biochimica et Biophysica Acta (BBA)-General Subjects 2014, 1840(5):1468-1481. 
28. Zargar SM, Nagar P, Deshmukh R, Nazir M, Wani AA, Masoodi KZ, Agrawal GK, Rakwal R: Aquaporins as potential drought tolerance inducing proteins: towards instigating stress tolerance. Journal of proteomics 2017, 169:233-238.

29. Bienert MD, Diehn TA, Richet N, Chaumont F, Bienert GP: Heterotetramerization of plant PIP1 and PIP2 aquaporins is an evolutionary ancient feature to guide PIP1 plasma membrane localization and function. Frontiers in plant science 2018, 9:382.

30. Lv X, Jin Y, Wang Y: De novo transcriptome assembly and identification of salt-responsive genes in sugar beet M14. Computational biology and chemistry 2018, 75:1-10.

31. Tournaire-Roux C, Sutka M, Javot H, Gout E, Gerbeau P, Luu D-T, Bligny R, Maurel C: Cytosolic pH regulates root water transport during anoxic stress through gating of aquaporins. Nature 2003, 425(6956):393-397.

32. Tamura K, Peterson D, Peterson N, Stecher G, Nei M, Kumar S: MEGA5: molecular evolutionary genetics analysis using maximum likelihood, evolutionary distance, and maximum parsimony methods. Molecular biology and evolution 2011, 28(10):2731-2739.

\section{Figures}


SD-Trp-Leu

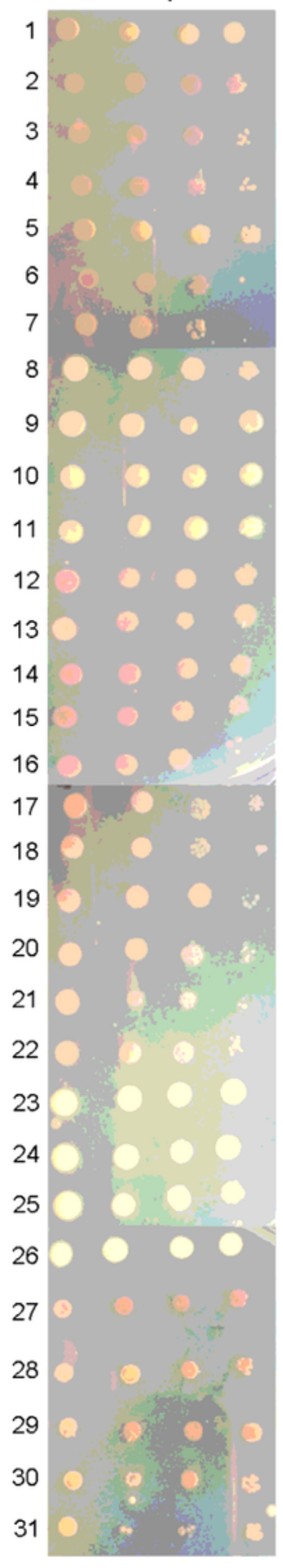

$1 \mathrm{x} 10 \mathrm{x} 100 \mathrm{x} 1000 \mathrm{x}$

\section{SD-Trp-Leu-Ade-His}

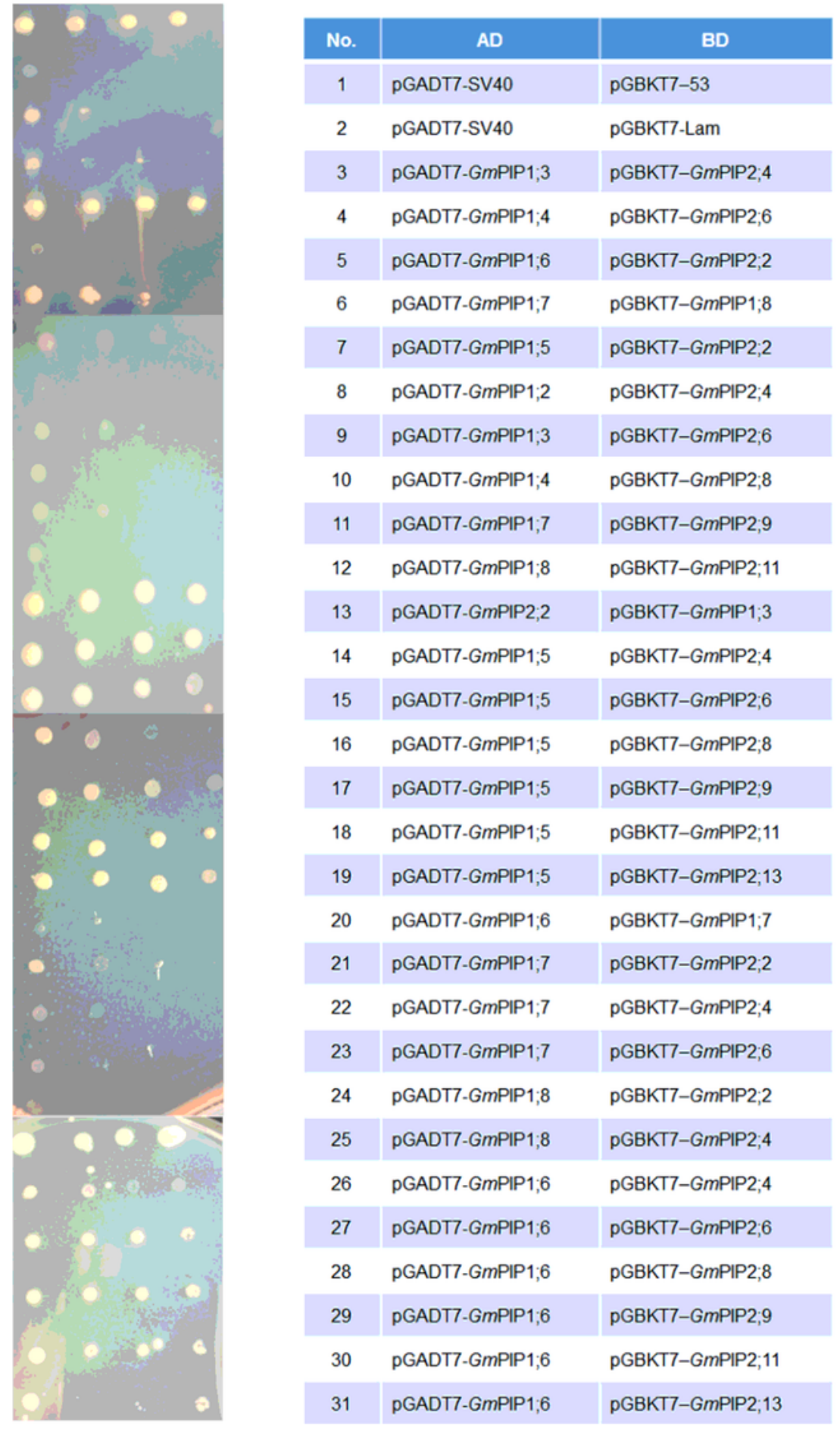

\section{Figure 1}

Yeast two-hybrid $(\mathrm{Y} 2 \mathrm{H})$ assays show GmPIP1s and GmPIP2s interact to form hetero-tetramers. Fulllength GmPIP genes were cloned into pGADT7 (with the activation domain, AD) and pGBKT7 (with the DNA-binding domain, BD). Varied combinations of pGADT7 and pGBKT7 constructs were co-transformed into the $\mathrm{Y} 187$ and $\mathrm{Y} 2 \mathrm{H}$ gold yeast strains. Yeast cells were grown in liquid selective medium until OD600 $=1.0$, and then spotted at 1-, 10-, 100-, and 1000-fold dilutions on the SD-Leu-Trp and SD-Leu-Trp-Ade-His 
selective solid-medium plates. They were put into the incubator with temperature at $30{ }^{\circ} \mathrm{C}$, until the colonies emerged. Yeast cells harboring pGBKT7-53/pGADT7-SV40 vectors were set as the positive control, and those with pGBKT7-Lam/pGADT7-SV40 the negative. The numbers 1-31 represent the AD and $\mathrm{BD}$ vectors listed in the table on the right.
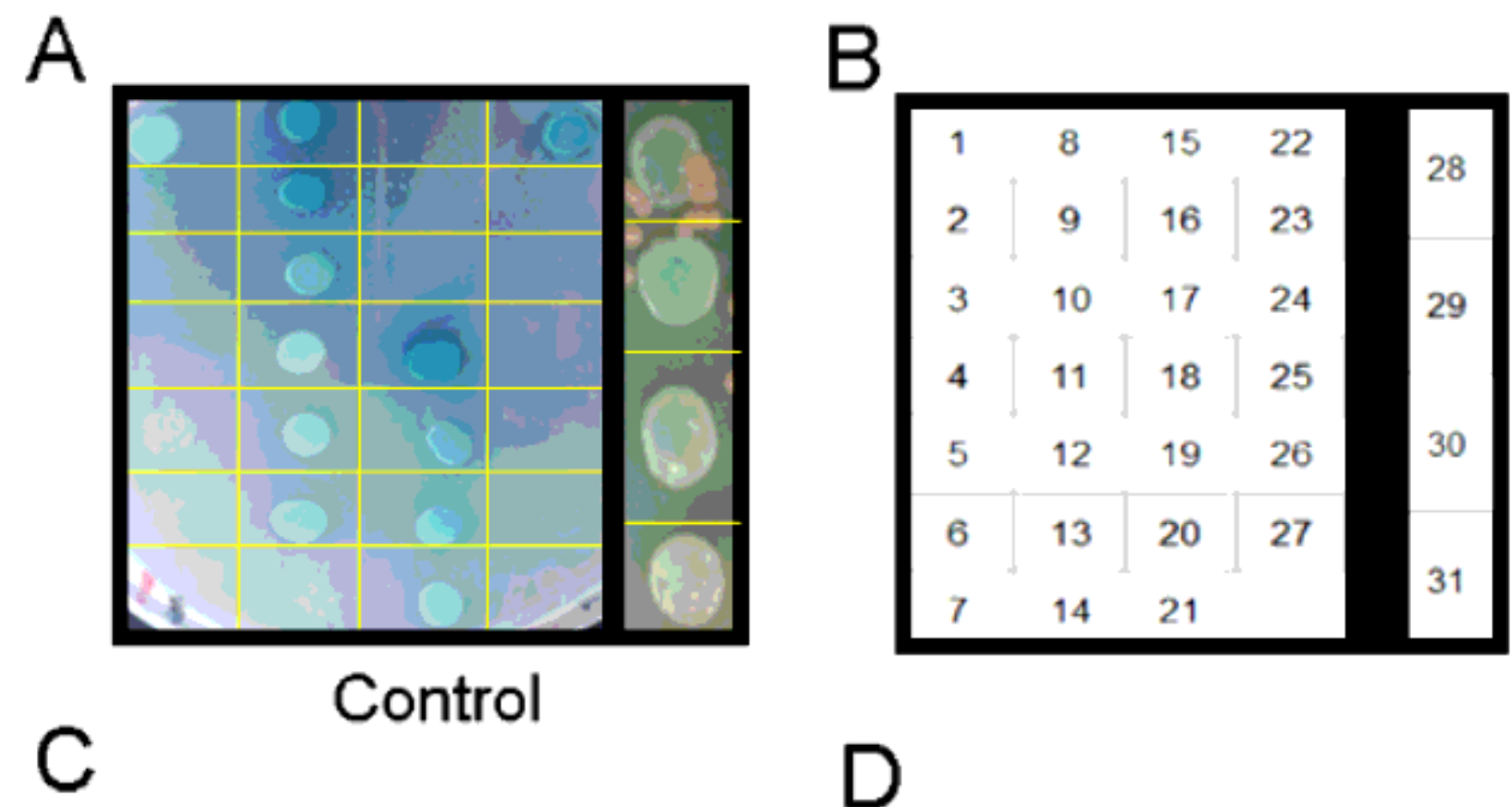

\section{Control}
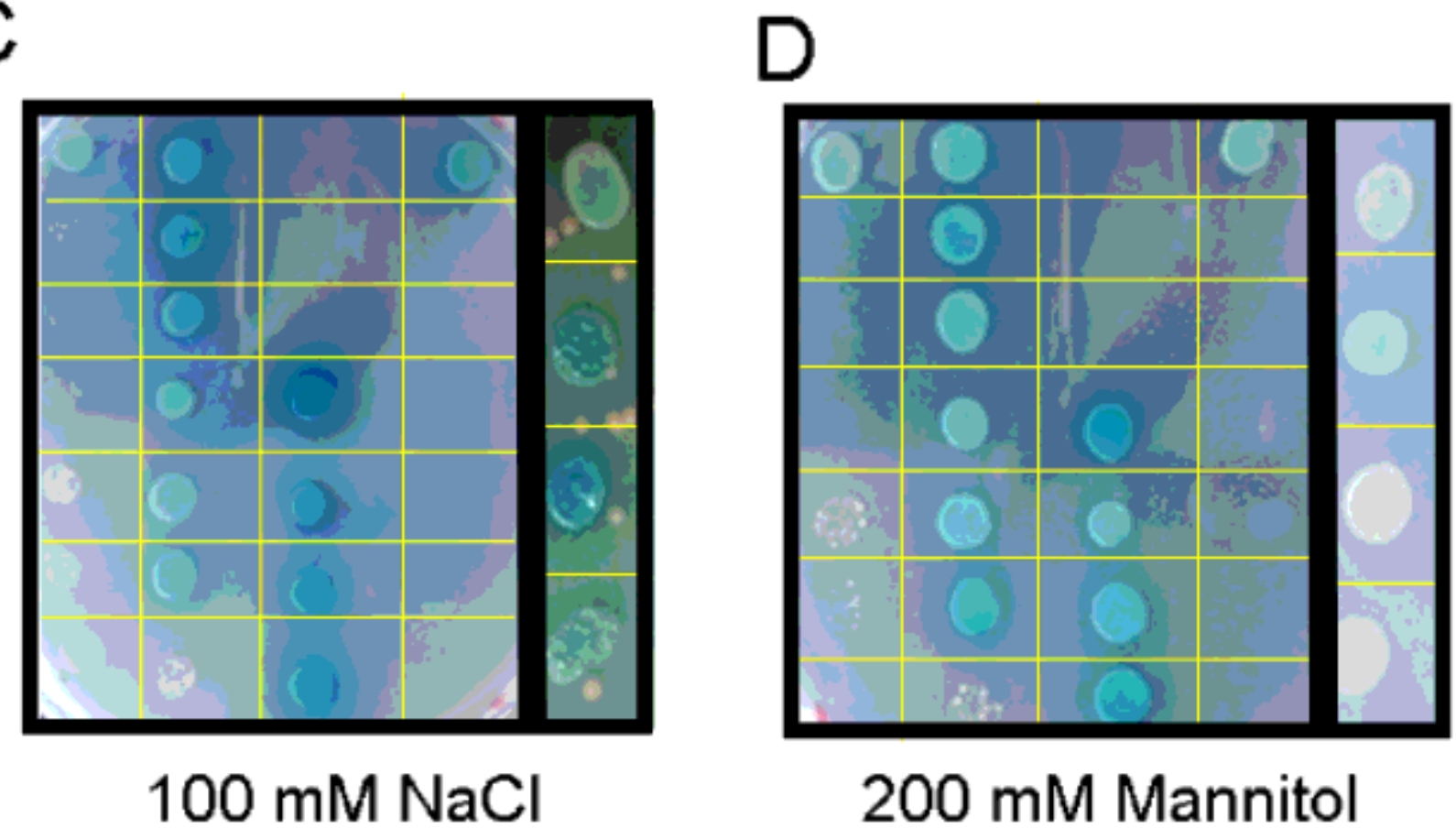

\section{0 mM Mannitol}

Figure 2

The effect of salt and osmotic stress on the interaction of GmPIP1s and GmPIP2s. (A) All the yeast clones mentioned in Figure 2 were plated on selective plates (SD-Leu-Trp-His-Ade) with Aba $(100 \mathrm{ng} / \mathrm{ml})$ and X-a-gal. (B) The position of the yeast clones on the plates. (C) The clones were spotted on the selective plates SD-Leu-Trp-His-Ade with Aba $(100 \mathrm{ng} / \mathrm{ml}), \mathrm{X}$-a-gal and $100 \mathrm{mM} \mathrm{NaCl}$. (D) The clones were spotted on the selective plates SD-Leu-Trp-His-Ade with Aba (100 ng/ml), X-a-gal, and $200 \mathrm{mM}$ mannitol. 


\section{SD-Trp-Leu}

GmPIP1;2
GmPIP1;3
GmPIP1;7
GmPIP1;8
GmPIP2;4
GmPIP2;9
GmPIP1;5
GmPIP1;6

GmPIP1;2
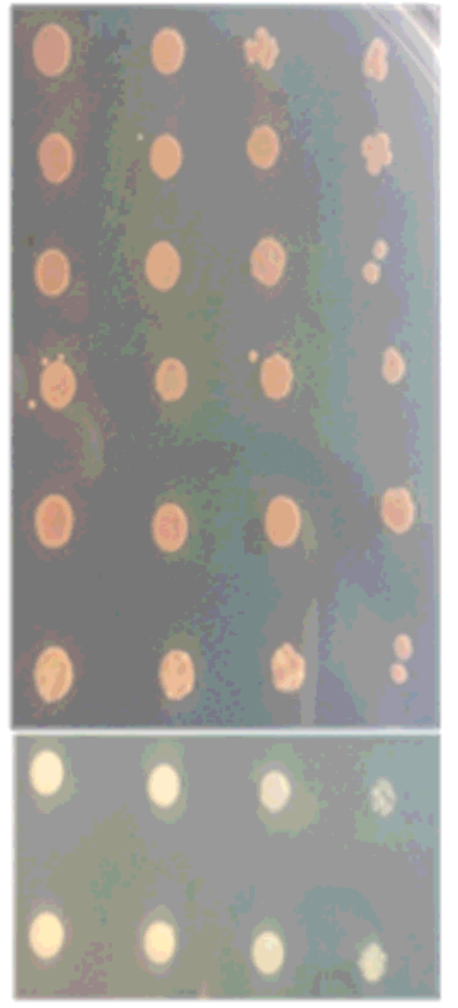

$1 \mathrm{X} 10 \mathrm{X} 100 \mathrm{x} 1000 \mathrm{X}$
SD-Trp-Leu-Ade-His (Aba+X-a-gal)
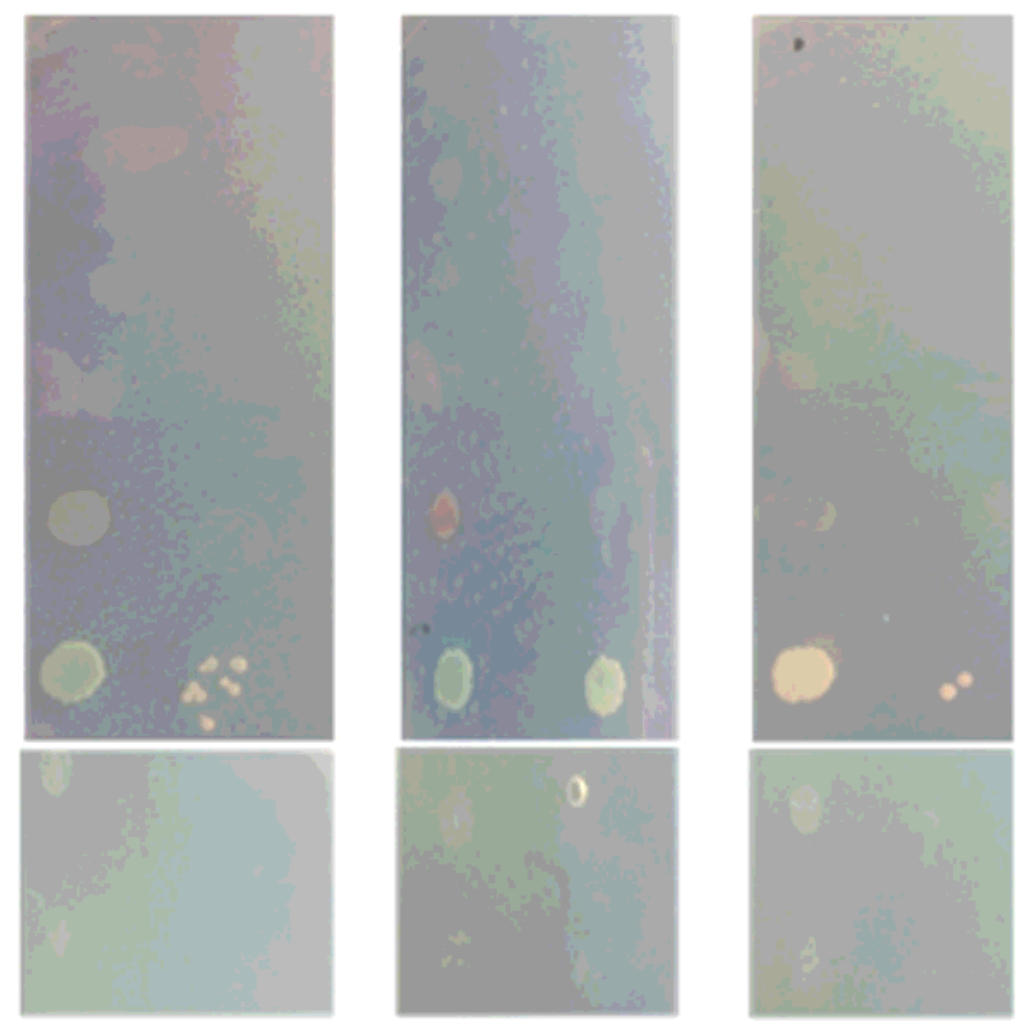

Normal

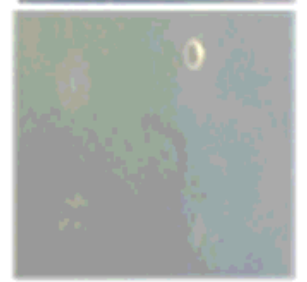

$\mathrm{NaCl}$

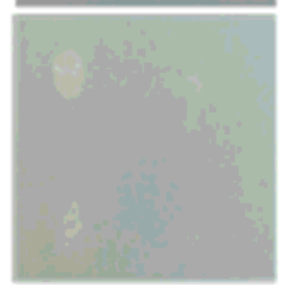

Mannitol

\section{Figure 3}

$\mathrm{Y} 2 \mathrm{H}$ to test homo-tetramerization of GmPIPs. Six GmPIP1 and two GmPIP2 genes were selected to construct the PGADT7 and pGBKT7 vectors. The same genes expressed in $A D$ and $B D$ were cotransformed into the $\mathrm{Y} 187$ and $\mathrm{Y} 2 \mathrm{H}$ gold yeast strain. The fresh yeast cells $(\mathrm{OD} 600=1.0)$ were spotted at 1, 10, 100 and 1000-fold dilutions media on the SD-Leu-Trp plates or SD-Leu-Trp-His-Ade+Aba (100 $\mathrm{ng} / \mathrm{ml}$ )+X-a-gal with $100 \mathrm{mM} \mathrm{NaCl}$ or $200 \mathrm{mM}$ mannitol 

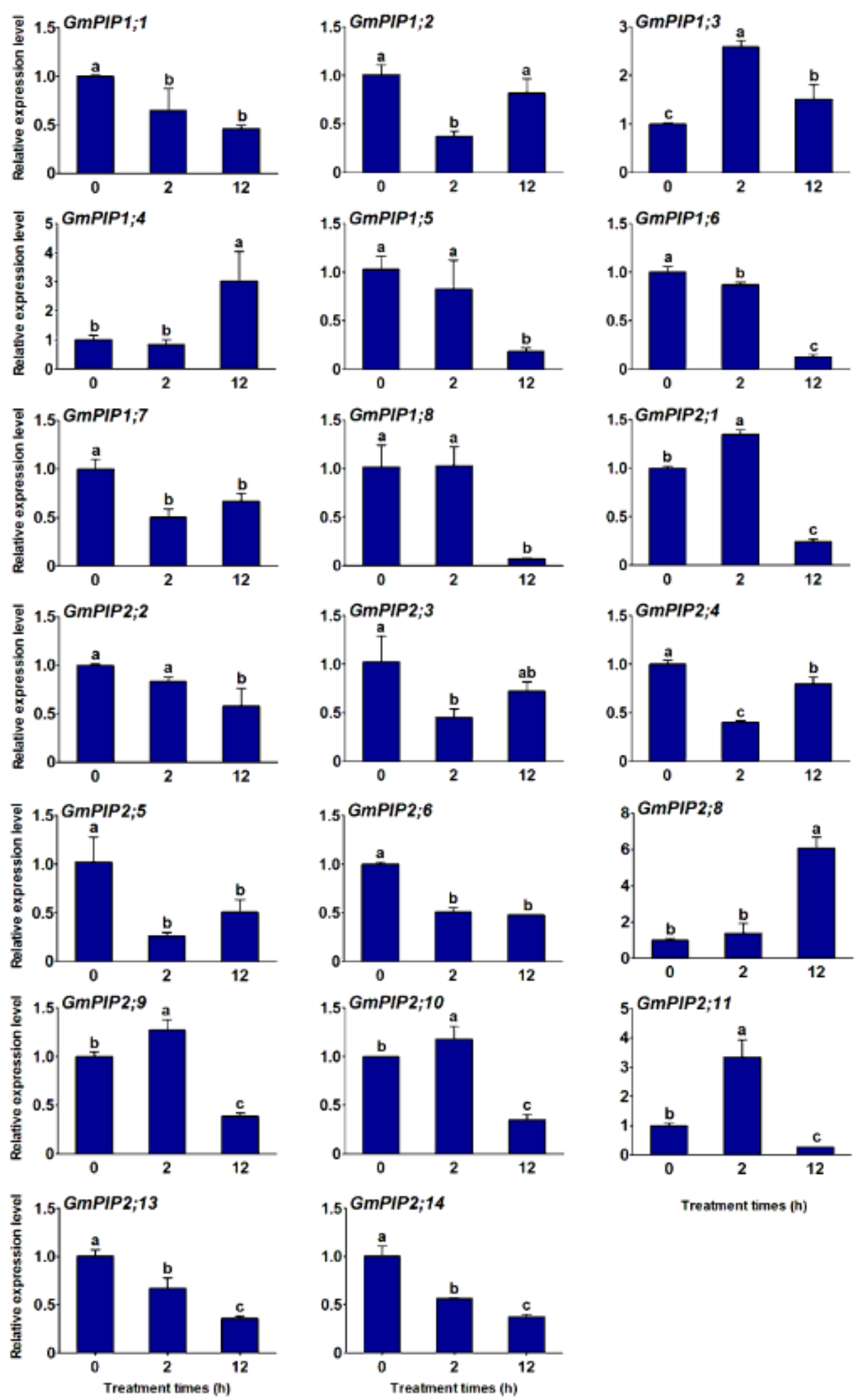

Treatment times (h)

Figure 4

Expression profile of GmPIPs in response to salt stress. The relative transcript levels of GmPIP genes were assayed by qRT-PCR, with soybean GmTubulin set as internal control. Roots of 2-week-old soybean seedlings were used to evaluate the changes of gene expression in response to salt stress $(200 \mu \mathrm{M} \mathrm{NaCl}$ for 0,2 , and $12 \mathrm{~h}$ ). The results are presented as means \pm SD of which three independent experiments were 
performed. Different alphabets indicate significant differences between controls and treated plants $(P<0.05$, one-way ANOVA).

\section{Supplementary Files}

This is a list of supplementary files associated with this preprint. Click to download.

- Additionalfiles.docx 